\title{
PAX8 is a novel marker for differentiating between various types of tumor, particularly ovarian epithelial carcinomas (Review)
}

\author{
LI XIANG and BEIHUA KONG
}

Department of Obstetrics and Gynecology, Qilu Hospital, Shandong University, Jinan 250012, P.R. China

Received November 28, 2012; Accepted January 3, 2013

DOI: 10.3892/ol.2013.1121

\begin{abstract}
Paired-box gene 8 (PAX8) encodes a transcription factor associated with important roles in embryogenesis and disease, and is a member of the PAX gene family. PAX8 has been demonstrated to be crucial in determining cell fate during the development of the thyroid, kidney, brain, eyes and Müllerian system and regulates expression of the Wilms' tumor suppressor gene (WT1). Several previous studies have reported that PAX8 is expressed at high levels in specific types of tumor, including thyroid and renal carcinomas and pancreatic neuroendocrine tumors. In addition, PAX8 has been reported to be useful for the detection and differential diagnosis of ovarian carcinoma. The consistency of PAX8 staining in epithelial ovarian carcinomas (EOCs) and the fallopian tube has provided morphological evidence that EOC may originate from the fallopian tube. The molecular mechanism of PAX8 in the carcinogenesis of these tumors remains unclear and requires further studies.
\end{abstract}

\section{Contents}

1. Introduction

2. Expression of PAX8 in various types of tumor

3. PAX8 detection in ovarian epithelial cancer

4. Conclusions

\section{Introduction}

Paired-box genes (PAX) encode a family of nine well-characterized paired-box transcription factors (PAX1-9), which are important for embryogenesis and disease (1). PAX proteins have been implicated as regulators of organogenesis and key factors in maintaining pluripotency of stem cell popula-

Correspondence to: Professor Beihua Kong, Qilu Hospital, Shandong University, 107 W. Wenhua Road, Jinan 250012, P.R. China

E-mail: kongbeihua@yahoo.com.cn

Key words: PAX8, tumor marker, ovarian epithelial carcinoma tions during development. Among these PAX genes, PAX8 has been hypothesized to be crucial in determining cell fate during the development of the thyroid, kidney, brain, eyes and Müllerian system and regulates expression of the Wilms' tumor suppressor gene (WT1) (2-7). A number of previous studies have identified that PAX8 is not only expressed in the aforementioned organs but also found at high levels in specific types of tumor, including thyroid and renal carcinomas and pancreatic neuroendocrine tumors (8-11). In addition, PAX8 has been found to be expressed at high levels in ovarian carcinomas and serous effusions $(3,12)$, indicating that PAX8 detection may prove useful in the clinic (Table I).

\section{Expression of PAX8 in various types of tumor}

A number of studies have found that PAX8 is important for the development of various types of tumor (8-10). Together with thyroid transcription factor (TTF)-1 and TTF-2 (FoxE1), PAX8 is markedly associated with thyroid gland organogenesis. PAX8 expression has also been identified in thyroid carcinomas $(10,13,14)$. Tacha et al found that PAX8 was expressed in $90 \%$ of thyroid cancer cases (15). In addition, follicular thyroid carcinoma (FTC) accounts for $10-20 \%$ of all thyroid cancers and up to $40 \%$ of cause-specific mortalities. Notably, the resulting fusion protein, PAX8/peroxisome proliferator-activated receptor (PPAR)- $\gamma$, has been found in $\sim 50 \%$ of FTC cases $(13,16,17)$. Chromosomal translocations involving PAX5 and PAX8 genes in thyroid cancer indicate that PAX genes have an oncogenic capacity when constitutively expressed, either as part of a fusion gene or as a whole gene $(10,18)$.

Previously, PAX8 was found to be markedly associated with renal tumors $(19,20)$. Renal cell carcinomas (RCC) stained positive for PAX8 in $90 \%$ of the cases studied and $100 \%$ of normal kidney samples stained PAX8-positive (15). Knoepp et al reported that immunoreactivity for PAX8 and PAX2 was observed in 88 and $83 \%$ of 24 cytology specimens, respectively. The presence of either PAX8 or PAX2 immunostaining revealed a total sensitivity of $92 \%$, indicating that PAX8 and PAX2 are useful adjuncts for confirmation of RCC diagnoses in cytology specimens (9). Collecting duct carcinoma (CDC) is a relatively rare but aggressive form of renal malignancy with variable morphological features and lacks a suitable marker for detection. A previous study found that all CDC cases were positive for PAX8. The immunoprofile 
of $\mathrm{PAX}^{+} / \mathrm{p} 63^{-}$is consistent with the diagnosis of CDC with a sensitivity of $85.7 \%$ and specificity of $100 \%$. By contrast, a $\mathrm{PAX} 8 \% \mathrm{p}^{+}$profile indicates upper urinary tract transitional cell carcinoma (UUC) with a sensitivity of $88.2 \%$ and specificity of $100 \%$. The nephric lineage restriction of PAX8 indicates a renal tubular rather than an urothelial differentiation in CDC according to the inverse PAX8/p63 expression observed in CDC and UUC (20).

Furthermore, PAX8 has been recognized as a potential immunohistochemical marker of pancreatic neuroendocrine tumors $(8,21)$. It was reported that among well-differentiated neuroendocrine tumors, only tumors from the pancreas were PAX8-positive $(14 / 25,56 \%)$ whereas no cases of pulmonary, ileal, duodenal, rectal or ovarian well-differentiated neuroendocrine tumors were positive for PAX8 (8). The observation that pancreatic well-differentiated neuroendocrine tumors frequently express PAX8 may be useful for distinguishing pancreatic primary tumors from tumors of other anatomical sites. By contrast, PAX8 expression is not specific for pancreatic origin in poorly differentiated neuroendocrine carcinomas, whereas it is observed in extrapancreatic poorly differentiated neuroendocrine carcinomas, indicating that PAX8 expression is not markedly associated with poorly differentiated neuroendocrine carcinomas. Sangoi et al found that PAX8 was positive in $74 \%$ of primary pancreatic neuroendocrine tumors and PAX8 expression did not correlate with World Health Organization categorization, grade, size, functional status or the presence of liver or lymph node metastases (22). Among liver metastases, only pancreatic neuroendocrine tumors $(20 / 31,65 \%)$ were PAX8-positive, whereas no cases of ileal, pulmonary, duodenal and rectal neuroendocrine tumor metastases were PAX8-positive. Overall, PAX8 is expressed in primary and metastatic pancreatic well-differentiated neuroendocrine tumors, enabling reliable differentiation between pancreatic and ileal and pulmonary well-differentiated neuroendocrine tumors using immunostaining methods.

Ovarian cancer is one of the most lethal forms of cancer in females and currently lacks useful markers and efficient screening methods (23), due to the complexity of variable subtypes. At present, the most common marker used in monitoring therapy of this disease is CA-125; however, this marker is not specific and sensitive enough to be useful as a screening test, with serum values in the normal range in half of patients with stage I disease $(24,25)$. Considerable efforts have been made to identify suitable markers for detection and differentiation between various forms of ovarian cancer. In 2003, analysis of the PAX8 gene by DNA microarray revealed high expression in ovarian cancer. Using the prediction analysis of microarrays (PAM) method, the expression of 61 genes was analyzed in 68 breast and 57 ovarian carcinoma samples and PAX8 expression was found at higher levels in ovarian compared with breast cancer (26). Consistent with these observations, microarray analysis performed by Bowen et al found that PAX8 was highly expressed in EOCs, whereas it was absent from the ovarian surface epithelia of healthy individuals. In addition, the authors observed that PAX8 was localized to the nucleus of non-ciliated epithelia in simple ovarian epithelial inclusion cysts and in 3 epithelial ovarian cancer subtypes (serous, endometrioid and clear cell). PAX8
Table I. Immunohistochemical expression of PAX8 in various forms of carcinoma.

\begin{tabular}{lcc}
\hline Histotype & $\%$ positive & Refs. \\
\hline $\begin{array}{l}\text { Thyroid carcinoma } \\
\text { Renal cell carcinoma }\end{array}$ & $79-90$ & $10,13,14,15$ \\
$\begin{array}{l}\text { Pancreatic } \\
\text { neuroendocrine tumors }\end{array}$ & $58-100$ & $9,15,19,20$ \\
$\begin{array}{l}\text { Ovarian carcinoma } \\
\text { Primary } \\
\text { Metastatic }\end{array}$ & $79-100$ & $8,21,22$ \\
\hline
\end{tabular}

PAX8, paired-box gene 8 .

was also found to be expressed in the non-ciliated secretory cells of healthy fallopian tube mucosal linings but not in the adjacent ciliated epithelia (27). Laury et al reported that PAX8 staining was present in $99 \%$ of high-grade serous ovarian carcinomas and $100 \%$ of low-grade ovarian carcinomas and serous borderline tumors (28). Tacha et al identified that $79 \%$ of ovarian cancers expressed PAX8 (15). These observations indicate that PAX8 may prove useful for the detection of ovarian cancers.

\section{PAX8 detection in ovarian epithelial cancer}

Differential diagnosis of ovarian and breast cancer. The ovary is a common site for formation of metastases and the breast is one of the most common sources. Ovarian and breast cancers develop from hormonally responsive tissues, comprise various histopathological subtypes and exhibit considerable variability in clinical manifestations and prognosis. Metastatic breast carcinoma is known to morphologically mimic primary ovarian carcinoma, resulting in difficulty in distinguishing between these forms of cancer. A previous study using microarray analysis revealed that PAX8 and EPAC are expressed at higher levels in ovarian compared with breast cancer (26). Previously, WT1 was considered to be a suitable marker to distinguish metastatic breast cancer from ovarian carcinoma. However, WT1 was later observed in focal breast cancer, causing false positive results. By contrast, PAX8 was stained in none of the breast and almost all ovarian cancer samples, indicating that PAX8 is a more superior marker for the differential diagnosis of ovarian and breast cancer (11).

Detection of metastatic ovarian carcinoma. Ovarian cancers are frequently associated with metastases, which are commonly found in peritoneal fluids (29). However, reactive mesothelial cells in effusion specimens are known to morphologically mimic ovarian serous carcinoma, making diagnosis difficult (30). Previously, calretinin was identified as a reliable immunohistochemical marker for mesothelial cells and WT1 was hypothesized to be useful in the diagnosis of ovarian serous carcinoma. However, mesothelial cells have also been found to exhibit immunoreactivity against 
WT1 (31). Recently, PAX8 was revealed to be expressed at high levels in EOC. By contrast, mesothelial cells stained negative against PAX8 (40). PAX8-positive, calretinin-negative staining appears to be highly specific and sensitive for detecting metastatic ovarian serous carcinoma in cytological preparations and may prove useful for distinguishing these cells from mesothelial cells in fluid cytology (32). Tong et al reported that PAX8 was detected in 70 and $68.8 \%$ of metastatic carcinomas of the ovary and endometrium in serous effusions, respectively (12). In addition, our previous study found that detection of PAX8 is useful for recognition of metastatic carcinomas in pelvic washings, particularly in cases with suspicious cytology (33).

EOC originates from the fallopian tube. EOC is one of the most common forms of ovarian cancer and its etiology and origin have been studied for a number of years. A number of hypotheses on the origin of EOC have been presented; however, none of these mechanisms have been officially recognized. A traditional hypothesis on the origin of EOC indicates that EOCs arise from the single layer of cells found surrounding the ovary, referred to as ovarian surface epithelia (OSE), which are the modified coelomic or peritoneal mesothelia that form a single layer of flat-to-cuboidal cells covering the ovary $(15,34,35)$. By contrast, additional studies have reported that EOC originates from the fallopian tube (36-39). Bowen et al observed that PAX8 was localized to the nucleus of non-ciliated epithelia in simple ovarian epithelial inclusion cysts and in three epithelial ovarian cancer subtypes (serous, endometrioid and clear cell). The authors found that PAX8 was also expressed in the non-ciliated, secretory cells of healthy fallopian tube mucosal linings but not in the adjacent ciliated epithelia (27), consistent with our own study (40). The findings suggested a possible correlation among EOCs, OSE and the fallopian tube. Marquez et al previously reported that when compared with normal ovarian epithelial brushings, alterations in microarray gene expression profiles of serous tumors correlated with those in normal fallopian tube $(\mathrm{P}=0.0042)$ but not in other normal tissues (41), indicating that EOCs not only imitate the phenotype of other differentiated epithelia of the female reproductive tract, but also their gene expression profiles. Morphological and genetic analyses indicate that expression levels of PAX8 are consistent with the hypothesis that EOC originates from the fallopian tube. At present, these observations remain controversial and challenging and additional studies should be performed to clarify the molecular mechanisms of PAX in the origin and carcinogenesis of EOC.

\section{Conclusions}

PAX8 is not only crucial for determining cell fate during the development of the thyroid, kidney and Müllerian system, but has also been found to be expressed at high levels in thyroid and renal carcinomas and pancreatic neuroendocrine tumors. Recently, PAX8 has been used in the detection and differential diagnosis of ovarian epithelial carcinomas. Consistencies in PAX8 staining between EOC and the fallopian tube indicate that EOC may originate from the fallopian tube. However, the molecular mechanism of PAX8 in the carcinogenesis of these tumors remains unclear and requires further analysis.

\section{References}

1. Muratovska A, Zhou C, He S, Goodyer P and Eccles MR: Paired-Box genes are frequently expressed in cancer and often required for cancer cell survival. Oncogene 22: 7989-7997, 2003.

2. Siehl JM, Thiel E, Heufelder K, et al: Possible regulation of Wilms' tumour gene 1 (WT1) expression by the paired box genes PAX2 and PAX8 and by the haematopoietic transcription factor GATA-1 in human acute myeloid leukaemias. Br J Haematol 123: 235-242, 2003.

3. Nonaka D, Chiriboga L and Soslow RA: Expression of pax8 as a useful marker in distinguishing ovarian carcinomas from mammary carcinomas. Am J Surg Pathol 32: 1566-1571, 2008.

4. Poleev A, Wendler F, Fickenscher H, et al: Distinct functional properties of three human paired-box-protein, PAX8, isoforms generated by alternative splicing in thyroid, kidney and Wilms' tumors. Eur J Biochem 228: 899-911, 1995.

5. Mittag J, Winterhager E, Bauer K and Grummer R: Congenital hypothyroid female pax8-deficient mice are infertile despite thyroid hormone replacement therapy. Endocrinology 148: 719-725, 2007.

6. Macchia PE, Lapi P, Krude H, et al: PAX8 mutations associated with congenital hypothyroidism caused by thyroid dysgenesis. Nat Genet 19: 83-86, 1998.

7. Dehbi M and Pelletier J: PAX8-mediated activation of the wt1 tumor suppressor gene. Embo J 15: 4297-4306, 1996.

8. Haynes CM, Sangoi AR and Pai RK: PAX8 is expressed in pancreatic well-differentiated neuroendocrine tumors and in extrapancreatic poorly differentiated neuroendocrine carcinomas in fine-needle aspiration biopsy specimens. Cancer Cytopathol 119: 193-201, 2011

9. Knoepp SM, Kunju LP and Roh MH: Utility of PAX8 and PAX2 immunohistochemistry in the identification of renal cell carcinoma in diagnostic cytology. Diagn Cytopathol 40: 667-672, 2012.

10. Marques AR, Espadinha C, Catarino AL, et al: Expression of PAX8-PPAR gamma 1 rearrangements in both follicular thyroid carcinomas and adenomas. J Clin Endocrinol Metab 87: 3947-3952, 2002.

11. Nikiforov YE: Molecular diagnostics of thyroid tumors. Arch Pathol Lab Med 135: 569-577, 2011.

12. Tong GX, Devaraj K, Hamele-Bena D, et al: PAX8: A marker for carcinoma of Müllerian origin in serous effusions. Diagn Cytopathol 39: 562-566, 2011.

13. Nikiforova MN, Lynch RA, Biddinger PW, et al: RAS point mutations and PAX8-PPAR gamma rearrangement in thyroid tumors: evidence for distinct molecular pathways in thyroid follicular carcinoma. J Clin Endocrinol Metab 88: 2318-2326, 2003.

14. Nonaka D, Tang $\mathrm{Y}$, Chiriboga L, Rivera $\mathrm{M}$ and Ghossein R: Diagnostic utility of thyroid transcription factors Pax8 and TTF-2 (FoxE1) in thyroid epithelial neoplasms. Mod Pathol 21: 192-200, 2008.

15. Tacha D, Zhou D and Cheng L: Expression of PAX8 in normal and neoplastic tissues: a comprehensive immunohistochemical study. Appl Immunohistochem Mol Morphol 19: 293-299, 2011.

16. Kroll TG, Sarraf P, Pecciarini L, et al: PAX8-PPARgamma 1 fusion in oncogene human thyroid carcinoma. Science 289: $1357-1360,2000$

17. Nikiforova MN, Biddinger PW, Caudill CM, Kroll TG and Nikiforov YE: PAX8-PPARgamma rearrangement in thyroid tumors: RT-PCR and immunohistochemical analyses. Am J Surg Pathol 26: 1016-1023, 2002.

18. Cazzaniga G, Daniotti M, Tosi S, et al: The paired box domain gene PAX5 is fused to ETV6/TEL in an acute lymphoblastic leukemia case. Cancer Res 61: 4666-4670, 2001.

19. Ozcan A, Shen SS, Hamilton C, et al: PAX 8 expression in non-neoplastic tissues, primary tumors and metastatic tumors: a comprehensive immunohistochemical study. Mod Pathol 24: 751-764, 2011.

20. Albadine R, Schultz L, Illei P, et al: PAX8 (+)/p63 (-) immunostaining pattern in renal collecting duct carcinoma (CDC): a useful immunoprofile in the differential diagnosis of CDC versus urothelial carcinoma of upper urinary tract. Am J Surg Pathol 34: 965-969, 2010.

21. Long KB, Srivastava A, Hirsch MS and Hornick JL: PAX8 expression in well-differentiated pancreatic endocrine tumors: correlation with clinicopathologic features and comparison with gastrointestinal and pulmonary carcinoid tumors. Am J Surg Pathol 34: 723-729, 2010 
22. Sangoi AR, Ohgami RS, Pai RK, Beck AH, McKenney JK and Pai RK: PAX8 expression reliably distinguishes pancreatic well-differentiated neuroendocrine tumors from ileal and pulmonary well-differentiated neuroendocrine tumors and pancreatic acinar cell carcinoma. Mod Pathol 24: 412-424, 2011

23. Jemal A, Center MM, DeSantis C and Ward EM: Global patterns of cancer incidence and mortality rates and trends. Cancer Epidemiol Biomarkers Prev 19: 1893-1907, 2010.

24. Nagele F, Petru E, Medl M, Kainz C, Graf AH and Sevelda P: Preoperative CA 125: an independent prognostic factor in patients with stage I epithelial ovarian cancer. Obstet Gynecol 86: 259-264, 1995 .

25. Eltabbakh GH, Yadav PR and Morgan A: Clinical picture of women with early stage ovarian cancer. Gynecol Oncol 75: 476-479, 1999.

26. Schaner ME, Ross DT, Ciaravino G, et al: Gene expression patterns in ovarian carcinomas. Mol Biol Cell 14: 4376-4386, 2003.

27. Bowen NJ, Logani S, Dickerson EB, et al: Emerging roles for PAX8 in ovarian cancer and endosalpingeal development. Gynecol Oncol 104: 331-337, 2007.

28. Laury AR, Hornick JL, Perets R, et al: PAX8 reliably distinguishes ovarian serous tumors from malignant mesothelioma. Am J Surg Pathol 34: 627-635, 2010.

29. Edge SB, Byrd DR, Compton CC, Fritz AG, Greene FL and Trotti A (eds): AJCC Cancer Staging Handbook. 7th edition. Springer, New York, NY, 2010.

30. Shivakumarswamy U, Arakeri SU, Karigowdar MH and Yelikar B. Diagnostic utility of the cell block method versus the conventional smear study in pleural fluid cytology. J Cytol 29: 11-15, 2012.

31. Bárcena $\mathrm{C}$ and Oliva E: WT1 expression in the female genital tract. Adv Anat Pathol 18: 454-465, 2011.

32. McKnight R, Cohen C and Siddiqui MT: Utility of paired box gene 8 (PAX8) expression in fluid and fine-needle aspiration cytology: an immunohistochemical study of metastatic ovarian serous carcinoma. Cancer Cytopathol 118: 298-302, 2010.
33. Xiang L, Zheng W and Kong B: Detection of PAX8 and p53 is beneficial in recognizing metastatic carcinomas in pelvic washings, especially in cases with suspicious cytology. Gynecol Oncol 127: 595-600, 2012.

34. Landen CN, Birrer MJ and Sood AK: Early events in the pathogenesis of epithelial ovarian cancer. J Clin Oncol 26: 995-1005, 2008.

35. Auersperg N, Wong AST, Choi KC, Kang SK and Leung PCK Ovarian surface epithelium: biology, endocrinology and pathology. Endocr Rev 22: 255-288, 2001.

36. Medeiros F, Muto MG, Lee Y, et al: The tubal fimbria is a preferred site for early adenocarcinoma in women with familial ovarian cancer syndrome. Am J Surg Pathol 30: 230-236, 2006.

37. Callahan MJ, Crum CP, Medeiros F, et al: Primary fallopian tube malignancies in BRCA-positive women undergoing surgery for ovarian cancer risk reduction. J Clin Oncol 25: 3985-3990, 2007.

38. Carcangiu ML, Radice P, Manoukian S, et al: Atypical epithelial proliferation in fallopian tubes in prophylactic salpingo-oophorectomy specimens from BRCA1 and BRCA2 germline mutation carriers. Int J Gynecol Pathol 23: 35-40, 2004.

39. Finch A, Shaw P, Rosen B, Murphy J, Narod SA and Colgan TJ: Clinical and pathologic findings of prophylactic salpingo-oophorectomies in 159 BRCA1 and BRCA2 carriers. Gynecol Oncol 100: 58-64, 2006.

40. Li J, Abushahin N, Pang S, et al: Tubal origin of 'ovarian' low-grade serous carcinoma. Mod Pathol 24: 1488-1499, 2011.

41. Marquez RT, Baggerly KA, Patterson AP, et al: Patterns of gene expression in different histotypes of epithelial ovarian cancer correlate with those in normal fallopian tube, endometrium and colon. Clin Cancer Res 11: 6116-6126, 2005. 